\title{
A THEOREM ON NILPOTENCY IN NEAR-RINGS
}

\author{
by S. D. SCOTT \\ (Received 12th October 1976)
}

Throughout this paper a near-ring $N$ will satisfy the distributive law $\alpha(\beta+\gamma)=$ $\alpha \beta+\alpha \gamma$ for all $\alpha, \beta$ and $\gamma$ in $N$. We shall also assume that $0 \alpha=0$ for all $\alpha$ in $N$.

We prove the following theorem.

Theorem. Let $N$ be a near-ring, $M$ a right $N$-subgroup of $N$ and $R$ a right ideal of $N$. If $M$ and $R$ are nilpotent, then so is $M+R$.

Proof. We first show that there exists a finite sequence

$$
N=T_{0} \geqslant T_{1} \geqslant T_{2} \geqslant \cdots \geqslant T_{k}=\{0\}
$$

of ideals of $N$, such that, $M T_{i} \subseteq T_{i+1}$ for $i=0, \ldots, k-1$.

Set $T_{0}=N$. For $i=1,2, \ldots$, define $T_{i}=T\left(M T_{i-1}\right)$, where $T\left(M T_{i-1}\right)$ denotes the ideal of $N$ generated by the subset $M T_{i-1}$ of $N$. It follows from the definition of $T_{i}$ that $M T_{i} \subseteq T_{i-1}$. Also $M T_{i} \subseteq T_{i}$, and thus $T_{i+1}=T\left(M T_{i}\right) \leqslant T_{i}$. It remains to prove that $T_{k}=\{0\}$ for some positive integer $k$. Take $k$ to be the smallest integer such that $M^{k}=\{0\}$. If $k=1$, then $M T_{0}=\{0\}=T\left(M T_{0}\right)=T_{1}$ and the result follows. Assume $k \geqslant 2$ and let $r$ be in $\{0, \ldots, k-1\}$. We shall show by induction on $r$ that $M^{k-r} T_{r}=\{0\}$. Since $M^{k} N=\{0\}$, the statement is true for $r=0$. If $M^{k-r+1} T_{r-1}=\{0\}$, then $M^{k-r} M T_{r-1}=\{0\}$ and $M T_{r-1} \subseteq\left(0: M^{k-r}\right)$. But $\left(0: M^{k-r}\right)$ is an ideal of $N$. Thus $T,=T\left(M T^{r-1}\right) \leqslant\left(0: M^{k-r}\right)$ and $M^{k-r} T_{r}=\{0\}$. In particular we have $M T_{k-1}=\{0\}$ and $T\left(M T_{k-1}\right)=T_{k}=\{0\}$.

Now suppose that $N=A_{0} \geqslant A_{1} \geqslant \cdots \geqslant A_{s}=\{0\}$ is a finite sequence of ideals of $N$ such that $M A_{i} \subseteq A_{i+1}$ for all $i$ in $\{0, \ldots, s-1\}$. Assume further that $s$ is minimal. We shall show by induction on $s$ that $M+R$ is nilpotent. If $s=1$, then $M N \subseteq A_{1}=\{0\}$. Hence

$$
(M+R)(M+R) \subseteq(M+R) N \subseteq R .
$$

If $R^{k}=\{0\}$ for some positive integer $k$, then

$$
(M+R)^{2 k}=\left[(M+R)^{2}\right]^{k} \subseteq R^{k}=\{0\}
$$

and $M+R$ is nilpotent. Suppose the theorem holds for $s-1$. Now $\left(M+A_{s-1}\right) / A_{s-1}$ and $\left(R+A_{s-1}\right) / A_{s-1}$ are nilpotent in $N / A_{s-1}$. Also

$$
\left(M+A_{s-1}\right) / A_{s-1} \cdot A_{i} / A_{s-1} \subseteq\left(M A_{i}+A_{s-1}\right) / A_{s-1} \subseteq\left(A_{i+1}+A_{s-1}\right) / A_{s-1}
$$

for $i=\{0, \ldots, s-1\}$. Thus $\left(M+A_{s-1}\right) / A_{s-1}$ has a finite sequence, as above, of length $s-1$. Since

$$
\left(M+R+A_{s-1}\right) / A_{s-1}=\left(M+A_{s-1}\right) / A_{s-1}+\left(R+A_{s-1}\right) / A_{s-1},
$$


we may assume that $\left(M+R+A_{s-1}\right) / A_{s-1}$ is nilpotent in $N / A_{s-1}$. Thus there exists a positive integer $p$ such that $(M+R)^{p} \subseteq A_{s-1}$. Now

$$
(M+R)^{p+1} \subseteq(M+R) A_{s-1} \subseteq M A_{s-1}+R .
$$

But $M A_{s-1} \subseteq A_{s}=\{0\}$ and thus $(M+R)^{p+1} \subseteq R$. Since $R^{k}=\{0\}$, it follows that $(M+$ $R)^{(p+1) k}=\{0\}$. Hence $M+R$ is nilpotent and the proof is complete.

Corollary. Let $N$ be a near-ring. A finite sum of nilpotent right ideals of $N$ is nilpotent.

For ideals the above corrollary is easily proved (see (1) or (2, Corollary 3.2)).

\section{REFERENCES}

(1) D. Ramakotaiah, Theory of near-rings, (Ph.D. dissertation, Andhra University, 1968).

(2) D. Ramakotalah, Radicals for near-rings, Math. Z. 97 (1967), 45-56.

10 Beacon Ave.,

CAMPBELls Bay, AUCKLAND,

NEW ZEALAND. 